\title{
EVOLIS Framework: a Method to Study Information Systems Evolution Records
}

\author{
Alexandre Métrailler \\ Faculty of Business and Economics \\ University of Lausanne, Switzerland \\ alexandre.metrailler@,unil.ch
}

\author{
Thibault Estier \\ Faculty of Business and Economics \\ University of Lausanne, Switzerland \\ thibault.estier@unil.ch
}

\begin{abstract}
This research proposes a framework that helps information systems managers to interpret present state of the system, to understand its past and to predict its future. The benefits of the framework are twofold. First, it gives managers a tool for assessing the impact of a change from either the users or the business/IS perspective. Managers can use this framework to design a strategy for information system evolution. Second, the framework allows the study of information systems evolution by revealing specific evolution patterns. In this context, this article presents a systematic method to inform components of this framework. The framework's structure is tested against six case studies on information systems evolution. Based on these case studies, this article presents a timeline of evolutions used to trace and study the life cycle of each information system according to the framework. Moreover, the case studies validate a trace analysis method and highlights specific evolution patterns.
\end{abstract}

\section{Introduction}

The rapid change occurring in business environments in response to evolving markets leads to a considerable amount of change in business processes. In order to cope with changes and new market opportunities, the information systems (IS) supporting these processes should be able to evolve in an adequate way.

This paper presents the conduct of case studies and their results in the context of our framework to study and to monitor IS evolution [1]. The paper is structured as follows. After this outline we present the field of IS evolution and the research question we want to address. Then in Section 2, we expose the research methodology used for this study, based on design science in IS. Section 3 presents the framework we are building and its constituting elements. Section 4 demonstrates the use of our framework. Section 5 presents the method used to conduct case studies and their results. Finally, the expected contributions and the future activities of this research are presented in Section 6 and 7.

The term evolution, in relation to software systems, has varying interpretations depending on stakeholders' view. In order to define evolution independently from subjective interpretations and following Lehman and Ramil [2], we consider IS evolution as a process of discrete progressive changes in architecture, workflows, features or functionalities of a system over time. For instance, an enterprise resource planning (ERP) system typically evolves by regularly adding new transactions, processes and views on business processes during its life cycle. M.M. Lehman [2] called such software systems the E-type systems (E for Evolving).

Several researchers described the evolution of Etype systems. To compare and categorize evolutions of such systems, Lientz and Swanson [4] proposed a software maintenance typology that distinguishes among perfective, adaptive and corrective maintenance activities. More recently, Chapin et al. [5] refined this typology into 12 different types of software changes. Moreover, they distinguished whether these changes are categorized as software maintenance or evolution. This work categorizes software changes on the basis of their purpose (i.e. the why of software change). Buckley et al.[6] take a complementary view of the domain; indeed, they focus on technical aspects by creating a taxonomy (i.e. the when, where, what and how of software change). On another side, Lyytinen Newman [7] describe and analyze the dynamics of IS change. They propose a model that uses sociotechnical event sequences and their properties to explain how a change outcome emerged.

These results provide a strong basis to classify software evolution according to each dimension cited above but do not address the effects of evolutions. In fact, little research has been carried out as to the impact in terms of IS management. To shed light on this area, this research investigates the motivations and consequences of changes regarding variations in terms of alignment between the business and the IS, IS 
architecture through integration among components of the IS, technological opportunities, user acceptance and cost of IS.

Concretely IS managers have to lead and to manage the evolution of the IS of their organization. In order to accomplish this task, they need to identify and to monitor the elements triggering an evolution of the system and their consequences.

This study is part of a design science research in IS which aims to build a framework that helps information systems managers to interpret present state of the system, to understand its past and to predict its future. Moreover, this framework allows the study of information systems evolution by revealing, specific evolution patterns.

This study addresses the following research question: "How a structured model like the EVOLIS framework can help revealing specific patterns in IS evolutions records?". Consequently we present a specific methodology developed to apply our framework in a posteriori case studies and the results obtained.

\section{Research methodology}

According to Hevner et al. [8], design science "creates and evaluates IT artifacts intended to solve identified organizational problems". In order to perform it, the research process follows the general design research cycle described by Kuechler and Vaishnavi [9].

A design science research in IS must fit in the framework described by Hevner et al. [8] and must follow their seven guidelines $(\mathrm{G})$ to conduct a design science research. The design science research produces artifacts, in our case a conceptual framework (named EVOLIS) (G 1), which must be relevant to a given business problem (G 2). The artifact must yield utility and then must be evaluated (G 3). The design science research must provide clear contributions in the areas of the design artifact $(\mathrm{G} 4)$. This research must rely on the application of rigorous methods for the creation and the evaluation of the artifact (G 5). In design science, the search process is inherently iterative whereby the search for an effective artifact requires using available means while satisfying laws in the environment (G 6). Finally, the design science research must be communicated to both technical and management audiences (G 7).

Table 1. Activities undertaken to follow Hevner et al. Guidelines [8]

\begin{tabular}{|c|l|}
\hline $\begin{array}{c}\text { Hevner et al. } \\
\text { Guidelines }\end{array}$ & \multicolumn{1}{c|}{ Activities of this research } \\
\hline $\begin{array}{c}\text { 1) Design as an } \\
\text { artifact }\end{array}$ & $\begin{array}{l}\text { The design of a framework to study IS } \\
\text { evolution }\end{array}$ \\
\hline
\end{tabular}

\begin{tabular}{|c|c|}
\hline $\begin{array}{l}\text { 2) Problem } \\
\text { relevance }\end{array}$ & $\begin{array}{l}\text { Relevance underlined by previous } \\
\text { studies on maintenance and evolution } \\
\text { management. }\end{array}$ \\
\hline $\begin{array}{l}\text { 3) Design } \\
\text { evaluation }\end{array}$ & $\begin{array}{l}\text { Observational evaluation by } \\
\text { conducting case studies for the } \\
\text { framework application }\end{array}$ \\
\hline $\begin{array}{l}\text { 4) Research } \\
\text { contributions }\end{array}$ & $\begin{array}{l}\text { It helps researchers to study IS } \\
\text { evolutions and practitioners to } \\
\text { manage and understand evolutions }\end{array}$ \\
\hline 5) Research Rigor & $\begin{array}{l}\text { Rigorous methods in both } \\
\text { construction and evaluation. }\end{array}$ \\
\hline $\begin{array}{l}\text { 6) Design as a } \\
\text { search process }\end{array}$ & $\begin{array}{l}\text { The design of the framework is an } \\
\text { iterative process. }\end{array}$ \\
\hline $\begin{array}{l}\text { 7) Communication } \\
\text { of Research }\end{array}$ & $\begin{array}{l}\text { Research must be presented to related } \\
\text { audiences }\end{array}$ \\
\hline
\end{tabular}

To design the framework, we rely on existing literature and on practitioners' feedbacks. The development and refinement of the framework is based on case studies and practitioners' interviews to refine and demonstrate the use of this framework. The evaluation of EVOLIS is qualitative, principally based on case studies, practitioners' feedback and satisfaction surveys. In further research, we will determine whether IS managers are willing to adopt the EVOLIS framework to evaluate the evolution of their IS and to use it as part of their IS strategy.

Currently, we conduct a first set of exploratory case studies to demonstrate the use of the framework and to refine it. According to Benbasat [10], case studies must be conducted when the researcher has less a priori knowledge of what are the variables of interest and how they will be measured. To demonstrate the use of the framework, we study the evolution of six information systems in six Swiss small and mediumsized enterprises (SMEs). Each system has evolved among two and four times. The sections 4 and 5 detail the methodology used to investigate the cases, the research on which we rely to classify evolutions and the results obtained.

\section{The EVOLIS Framework}

EVOLution of IS (EVOLIS) will invariably occur and can be caused by a large variety of factors: bugs that needs to be fixed, users that wish to have new functionalities, new market opportunities that require new software features, performance standards that the system must reach, technical changes in the environment with which the system must interact, obsolescence of applications, and so on. To face these evolutions managers in charge of IS have to make choices, for example to prioritize changes, deployments and projects.

Let us consider an example: in order to address an upcoming business need, an organization understands 
that a specific component of the IS (an application server for instance) will need to interact with another system outside of the company. So, the main motivation for this evolution is the alignment of the IS with the business. Such an evolution frequently contains activities like: studying specific users needs, studying the software system outside the company, implementing an interface between the two systems, testing the integration of both systems, evaluating a new end-users interface, training the users, updating documentation, etc...

The manager in charge of this project may want to evaluate and to quantify the impact of this evolution on several IS aspects. He/She may use the EVOLIS framework to identify all the factors and aspects of this change.

After discussions with experts, we concluded that the framework should answer to the following specifications:

- It is understandable and usable for managers.

- It addresses users' perceptions of the system and their efficiency using the system.

- It takes into consideration objective factors such as the maturity level of technology used, the alignment of the solution with the business and its level of integration, thus how well the IS support the business.

- It reports the overall cost of the IS.

The second usage of this framework is the study of IS evolution a posteriori. It enables the researcher or the manager to retrace the life cycle, and thereby, to locate the IS in its life cycle. According to these indications, the use of EVOLIS also helps to design the strategy of IS evolution. The framework acts as a dashboard of indicators to determine in which direction the system should evolve.

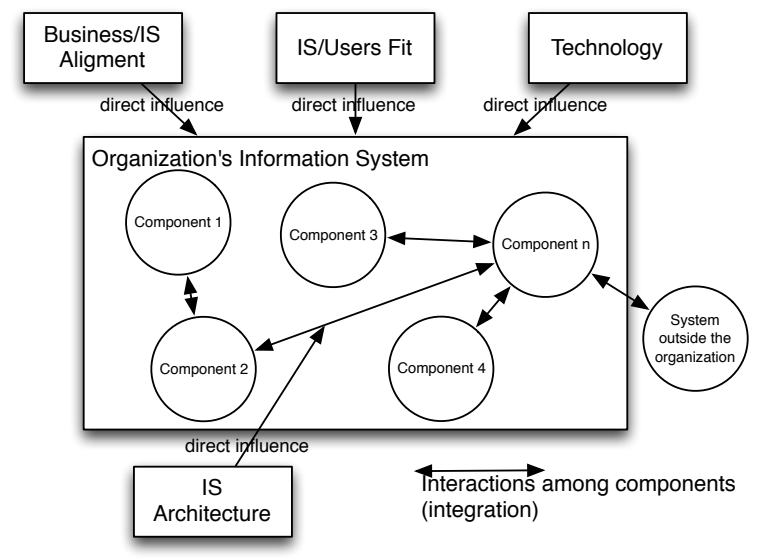

Figure 1. Factors influencing IS evolution represented in the EVOLIS framework.
The EVOLIS framework can be depicted as a canvas consisting of 4 blocks representing factors having a direct influence on the IS (illustrated in Figure 1): IS/Users Fit, Technology, IS Architecture including integration of IS components and IS Alignment with the business. Furthermore, the framework contains a fifth block related to the Cost of IS evolutions and maintenance.

\subsection{Business/IS Alignment}

This block addresses the co-alignment between business and information systems. To achieve a global and complete alignment of IS, Camponovo and Pigneur [11] suggest considering three levels of alignment. The first alignment level corresponds to the nowadays classical alignment of IS with business organization's goals and activities, namely its strategy, organization and technology infrastructure. The second level takes into consideration the alignment with the external environment of the organization and assumes that the IS has to integrate features for assessing this environment. The third level of alignment copes with the evolution over the time and points out the necessity to design and to adapt the IS with the purpose of being able to evolve according to future changes of the organization and its environment. Despite the classical alignment of IS with business operations and strategies remains the first step to succeed in alignment, the two other levels are crucial regarding the complexity and uncertainty of the business environment.

3.1.1 Strategic and operational alignment. Research has shown that IT-business strategic alignment contributes to higher levels of organizational performance [12]. Their study was designed on the Strategic Alignment Model (SAM) [13] which identifies four domains: business strategy, IS strategy, organizational infrastructure, and IT infrastructure (illustrated in Figure 2). The SAM identifies two types of alignment between business and IT domains. The first, termed strategic integration is the link between business strategy and IT strategy. It addresses the capability of IT functionality to support a business strategy. The second type, termed operational integration deals with the organizational infrastructure and processes and IS infrastructure and processes. It address the coherence between the organizational requirements and expectations and the capabilities of the IS function. 


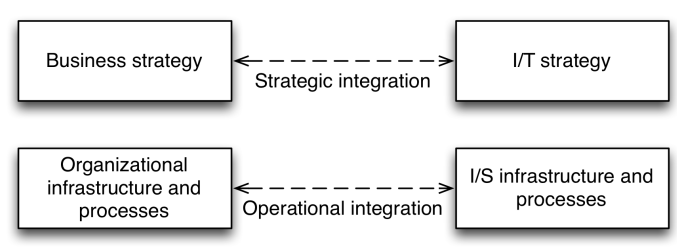

Figure 2. The major components of the Strategic Alignment Model [13].

The framework classifies IS evolutions under both operational and strategic perspectives. The purpose of the "operational alignment perspective" is to describe the evolution of the fit between business processes and IS processes. Evolutions are classified as "operational" when evolutions impact processes issue of support functionalities or when they correct processes issue of core business functionalities. In other words, the "operational alignment perspective" deals with corrections of the system in use.

The purpose of the "strategic alignment perspective" is to describe the evolution dealing with the scope of the IS, whether it is extended by the evolution and whether the evolution has a strategically perspective on core business functionalities or support functionalities.

3.1.2 External environment alignment. Assessing the environment in which an organization deploys their activity is crucial to ensure its continued business performance. Many well-known models as the SWOT model, the five forces model or the disruptive technology framework suggest that an organization must pay sufficient attention to its environment.

Therefore, the EVOLIS framework must take into account this alignment perspective. We consider an evolution as "external environment alignment" when it addresses features for assessing the organization environment.

3.1.3 Evolution oriented alignment. Evolution in the environment or organization's strategy and operational activities would require information systems to be aligned repeatedly.

In the case of rapidly changing environments, a well-known anticipation technique is to elaborate future evolution scenarios. These scenarios provide a mean to manage and to design information systems that take into account multiple evolution possibilities in advance. Thus these systems can remain aligned for a longer time.

An evolution is classified as "evolution oriented alignment" when the selection of a specific evolution is retained according to future business evolution scenarios.
Another interesting approach to study the alignment between business and IS is to focus on the dynamics of alignment. Sabherwal and Hirschheim [14] study how does the alignment evolve over time. They point out that the punctuated equilibrium model provides a good perspective for viewing the dynamics of alignment. This work on evolution of alignment is valuable for our research as we plan to observe not only a static alignment, but also the alignment over time.

\subsection{IS/Users Fit}

Users are important in terms of evolution; actually, many researchers mention that key incentives of evolution are feedbacks and change propositions coming from the end users [15]. The IS Fit with Users is measured using both subjective and objective approaches. The users' satisfaction with the IS is measured using the well known perceived usefulness and perceived ease of use (TAM [16]). These two variables determine whether users accept or reject an information technology. Consequently, these two variables enable managers to measure the perceived benefits or losses of a change. Islam et al. [17] propose a lightweight instrument to measure users' satisfaction and service quality experienced by the users. This instrument provides a great indication on the introduction of a new service and can be easily applied by managers to obtain feedback on IS evolutions.

On the other hand, to study a posteriori evolution records in our case studies, we classify as IS/User Fit each activity during an evolution regarding directly users or when the evolution only alters the fit between IS and users without altering business functionalities.

\subsection{IS Architecture}

The role of the IS architecture block in our framework is twofold, first the IS Architecture strategy of the company impact directly the way the IS will evolve, second it enables the manager to control variations of the IS scope after each evolution.

The integration among IS components influence directly the IS scope. Thus, we use the integration as a tool to assess and monitor the IS scope variations in terms of business functionalities.

The IS architecture block measures the delta related to IS scope between the past IS and the changed IS. The literature provides many frameworks related to enterprise application integration (EAI) like the Brown's Conceptual Model of Integration [18] and Zachman's Enterprise Architecture Framework [19].

In this study of a posteriori evolution records, we classify an IS Architecture evolution according to different types of integration evolution, namely an 
evolution of integration among components of the system, among business functionalities, or an integration with systems outside of the company. Consequently, this classification provides an evolutionary map of IS components and an accessible mean to control IS architecture.

\subsection{Technology}

When it comes to IS evolution, software and hardware platforms play a critical role. Costs incurring from using an inappropriate technology could be significantly increased whether a change is required. The Technology block encompasses notions like the degree of innovation, anticipation, flexibility, scalability, portability and so on of IS components. We classify an evolution as a technology evolution whether the trigger of this evolution is the technology itself. As example when reasons like performance, updates, preventive maintenance and so on motivate evolutions of the software or hardware.

\subsection{Cost}

The cost (value) of the IS is an important element to take into account when studying evolution: both academic and practitioners agree with the fact that IS investments should be carefully justified, measured and controlled. In practice, many traditional techniques are used to evaluate the "cost-benefits" of IS investments for example the Return On Investment (ROI), the Payback Period (PP), the Internal Rate of Return (IRR) and so on [20]. We believe that the four previous EVOLIS blocks must be evaluated in parallel with the cost function of the IS. We do not specify a particular technique to evaluate the cost-benefit ratio of evolutions. Nevertheless, we recommend not forgetting to include into the cost calculation.

In a research context, while making a posteriori measurements on evolution logs, detailed information on cost structure is difficult to reconstitute. Consequently, in the following case studies, we choose to replace it with the human energy involved in working days for each activity of an evolution. Information on effort in man/days or man/hours is often available and traceable in evolution logs, giving a very good cost indirect indicator.

\section{Framework application}

As mention previously, we follow the taxonomy described in [6] to describe an evolution. Since IS evolution or maintenance typically involves many activities, we identify an evolution as being the accomplishment of activities impacting the IS and followed by a "go live". In other words, we define an evolution as being a new release of the IS used in companies.

\subsection{Classification of evolutions}

The first step to classify the evolution of a company IS is to organize them chronologically. As a result of this classification, it becomes possible to retrace the life cycle of the IS with its evolutions. This position of the evolution in time answers to the taxonomy question when of evolution.

The second step is to identify the triggers of IS evolutions. So, for each evolution, we identify the main motivations that initiate this evolution. According to the framework, an evolution is classified as

- Business/IS alignment, whether the motivation of the evolution is an alignment of the IS with the business needs or the introduction of new business functionalities

- IS/Users fit, whether the relation between the IS and the users motivates the evolution

- Technology, whether the evolution is triggered by software or hardware motivations

- IS Architecture, whether the evolution refactors, integrates or consolidates existing functionalities within the IS

Obviously, an evolution may involve a mix of triggers, even though one framework criteria may be deemed as dominant.

Moreover, the knowledge of the cost of the evolution is important to determine its magnitude. The magnitude of an evolution is useful to rapidly evaluate its importance in the life cycle of the IS.

This classification of the evolution according to the five framework criteria corresponds to the taxonomy question why of evolution.

At this point, we can retrace the life cycle of the IS with its evolutions, their motivations and their importance.

\subsection{Classification of activities within evolutions}

The first step to classify activities is to identify the various activities. We define an activity as a task or set of tasks accomplished for a specific purpose. We draw upon prior work on the activities and tasks involved in doing software maintenance and evolution, and more specifically on the listing made by Chapin et al. [5]. By identifying the required activities required by an evolution, we answer to the taxonomy question how to evolve.

The activities involved by an evolution may result in none to many changes is the IS. Indeed, an activity 
can be namely "training of users" and have no direct effect on the IS implementation but these activities remain essential in the success of the evolution. To determine the type of each activity we follow the decision tree proposed by Chapin et al. [5] and presented in Figure 3. The proposed classification is composed by 12 mutually exclusive types grouped in 4 clusters.

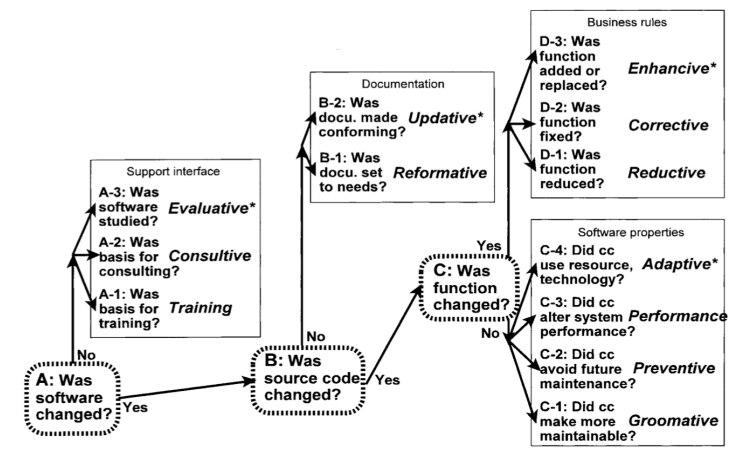

Figure 3. Chapin et al. [5] decision tree to determine type of evolution or maintenance activities

This classification of activities within an evolution points out a finer granularity view providing an insight and management opportunities. Managers are frequently interested in seeing how resources are spent for the maintenance and evolution of the information system.

After having shed light on the nature of each activity, it is interesting to clarify the purpose of each activity according to the EVOLIS framework. The aim of this classification is to determine the amount of resources allocated for each framework block in an evolution.

To demonstrate the framework application by practitioners, we describe a scenario based on the previous example of evolution activities $\left(2^{\text {nd }}\right.$ paragraph of Section 3).

Concretely in this scenario, the team members (technicians, business analysts, etc.) in charge of this project will repertories and classifies each previously mentioned activities according to Chapin et al. typology [5]. The weighting of each activity in the evolution is important to determine the resources allocation within the evolution. To assess the activities weighting, the team members will measure the time spend for each activity. After having shed light on the nature of these activities, one must determine the purpose of each activity according to the EVOLIS framework.

Immediately at the end of this IS evolution having for primary purpose the alignment of the IS with business needs (the interaction with a system outside of the company). The manager could determine the repartition of the resources involved in this evolution according to the EVOLIS framework. Namely in this evolution scenario, the majority of tasks required to align the IS have an architectural purpose related to the integration with a system outside of the company.

Activities such as studying the software system outside the company, implementing the communication between the two systems and testing the integration of both systems are related to the IS Architecture block of the framework because the IS architecture is at once impacted by the extension of the IS scope and the company IS architecture strategy determines the technique used to integrates the two systems.

Activities such as studying specific users needs, evaluating the new user interface or training the users are related to IS/Users Fit because the purpose of these activities impact the relation between IS and users.

The repeated use of the framework for each evolution of the IS and the study of activity classification could point out multiple managerial advantages.

As example, after having studied the repartition of activities, it could namely justify allocating more resources to activities classified as "Consultive" or "Evaluative" in order to save resources on other activities or on the global cost of an evolution.

\section{Case studies}

This section details the systematic method used to analyze a posteriori each IS evolution of the various case studies and presents some results.

A posteriori study of IS evolution is useful for both IS managers and researchers. Indeed, this study provides managers a better understanding of past evolutions and foundations on which they can rely to predict and justify investments. In addition to that, it helps managers to design the IS strategy of evolution. On the other hand, researchers can better understand the triggers of IS evolution during each sate of IS life cycle and a longitudinal view enables researchers to identify specific patterns of evolution.

\subsection{Presentation of the collected records}

In the first application of the EVOLIS framework on real evolution cases, we orient the selection of cases to small structures like applications of SMEs. Small companies with a modest sized IS seem more appropriate for a first exploratory study.

Despite the fact that we selected SMEs IS as case studies, we focus on companies having a higher degree of IS maturity than the simple use of advanced spreadsheet. So, we selected companies having at least 
already implemented an integrated operational platform (ERP). We define, as starting point of the evolution of each IS, the first change to the system since the end of the ERP deployment.

As the companies chosen for this case study are SMEs which main business is not IT, the development and maintenance of their IS is outsourced to specialists.

The records collected in this study are documents containing the detailed invoices made by their IT specialists. We have one set of documents per company studied. Each set of documents contains the chronological list of every interventions on the company IS and their corresponding duration. In all sets of documents, every intervention is followed by a "go live", thus, we consider an intervention as being an evolution. For each intervention on the IS, there is a description of its global purpose and a list of every specific activities of the intervention. Moreover, the documents contain the purpose, the reason, the duration for every activity and the detailed work done.

In order to respect a systematic method for addressing the study of IS evolutions, we followed the recommendations of Yin [21] to conduct case studies. Yin explains that case studies are appropriate to address the how and why questions rather than mere frequencies of incidence. Moreover, Yin and Paré and Dubé [22] support that the what question is frequently posed in exploratory case research. As the purpose of this research is to study IS evolutions over time, we address also the when question. To summarize, for each evolution we identify:

- When of evolution (because of the longitudinal design of our research)?

- Why of evolution (what triggers the evolution)?

- How of evolution (how the evolutions has been carried out)?

- What of evolution (what is the difference between before and after the evolution)?

In addition to these four questions, we notice the number of working days to apprehend the effort involved to accomplish the change and the magnitude of an evolution.

All the sources of these case studies rely on documents investigated a posteriori. Thus, we do not spend time on site during evolutions. For each of the six companies, a set of documents, sorted by evolution, is available. We define an evolution as being the accomplishment of activities followed by a "go live". As the documents are a mix between release notes and project planning activities, it is simple to determine the beginning and the end of a single evolution.

The documents contain a listing of the new features, their purpose, some reasons and the details of their implementation. Moreover, for each activity undertaken on a system, the documents contain the details of impacted features and the duration of each activity. We investigate each document according to the systematic method described previously.

The fist step of the investigation is to bring together all documents related to a single evolution. Once this classification is done, we carry out the following activities according to the taxonomy described in [6]:

1. Position the evolution in time: when of evolution

2. Identify the motivations triggering the evolution according to the framework: why of evolution

3. Notice the activities required for the evolution: how to evolve

4. Determine the type of each activity according to [5] : what is performed for the evolution

5. Notice purpose and motivation for each activity according to the framework: why of evolution

6. Evaluate the resources (working days) required for each activity: magnitude of evolution

The evaluation of the resources involved in an activity (through the working days) allows us to allocate a weighting to each activity for the whole evolution. As a result of this weighting, we establish a repartition of the different kinds of EVOLIS criteria addressed by one evolution.

\subsection{Case studies results}

We repeated this systematic document analysis to the 18 evolutions of the 6 companies IS. With this analysis, we are able to trace the evolution of each system and to point out where the resources are invested, namely in business/IS alignment, in architecture, and so on. Moreover, by observing the timeline of evolutions, we can determine in which state of its life cycle the system lies. Table 2 presents the repartition of working days allocated to each activity, arranged by company and by evolution chronologically. We extract these working days from the detailed work and fees justification that were in the invoices and documents made by the IT consultant for every intervention in the 6 companies.

The 6 companies are SME, despite the fact that they all use an ERP, the complexity of their IS remains low. Moreover, the companies are followed by the same IT specialists since the beginning, thus the specialists have a perfect knowledge of their IS. Furthermore the SMEs prioritize technologies recommended and mastered by their IT specialists. These facts explain the relative small amount of time spent for the majority of evolutions (the amount of 
work involved in 14 out of the 16 evolutions is one week or less)

All companies use their system since 4 years $(2009$ to 2012) or less. Based on that, we can make the hypothesis that a majority of systems should be in the first part of their life cycle during which the resources are invested in aligning and extending the system, rather than in migration of legacy systems.

Based on the resources repartition, namely the working days, allowed for each activity of each evolution, we compare chronologically each evolution for all companies, as example, we compare all the first evolutions among them regardless of the companies.

Table 2. Working days of an evolution allocate to each block of the framework

\begin{tabular}{|c|c|c|c|c|c|}
\hline Company A & & IS/Users Fit & $\mathrm{B} / \mathrm{IS}$ alignment & Technology & IS Architecture \\
\hline \multirow[t]{3}{*}{ Evolutions } & 1 & 0.5 & 4 & 0 & 0 \\
\hline & 2 & 1 & 0 & 0 & 3.5 \\
\hline & 3 & 1 & 1 & 0 & 3.5 \\
\hline \multicolumn{6}{|l|}{ Company B } \\
\hline \multirow[t]{2}{*}{ Evolutions } & 1 & 0 & 3.5 & 0 & 1 \\
\hline & 2 & 2 & 24.5 & 0 & 16 \\
\hline \multicolumn{6}{|l|}{ Company C } \\
\hline \multirow[t]{4}{*}{ Evolutions } & 1 & 0.3 & 1.5 & 0.2 & 0 \\
\hline & 2 & 1 & 1.5 & 0 & 2 \\
\hline & 3 & 1.5 & 3 & 0 & 1 \\
\hline & 4 & 1.5 & 0 & 0 & 3.5 \\
\hline \multicolumn{6}{|l|}{ Company D } \\
\hline \multirow[t]{4}{*}{ Evolutions } & 1 & 0.2 & 10.5 & 0 & 0.3 \\
\hline & 2 & 0.2 & 2 & 0 & 0.3 \\
\hline & 3 & 0.2 & 1.5 & 0 & 0.3 \\
\hline & 4 & 1.5 & 1 & 0 & 0.5 \\
\hline \multicolumn{6}{|l|}{ Company E } \\
\hline \multirow[t]{2}{*}{ Evolutions } & 1 & 0 & 3 & 0 & 0.5 \\
\hline & 2 & 1 & 3.5 & 0 & 2 \\
\hline \multicolumn{6}{|l|}{ Company F } \\
\hline \multirow[t]{3}{*}{ Evolutions } & 1 & 0.5 & 2 & 0 & 0.5 \\
\hline & 2 & 3 & 12.5 & 0 & 2 \\
\hline & 3 & 0 & 2.9 & 0 & 1.1 \\
\hline
\end{tabular}

Table 3. Percentage of working days allocate to each building block according to evolutions.

\begin{tabular}{|l|c|c|c|c|c|}
\cline { 2 - 6 } \multicolumn{1}{c|}{} & IS/Users Fit & B/IS alignment & Technology & IS Architecture \\
\cline { 2 - 6 } & 1 & $7.43 \%$ & $81.58 \%$ & $1.67 \%$ & $9.32 \%$ \\
\cline { 2 - 6 } & 2 & $14.95 \%$ & $49.38 \%$ & $0.00 \%$ & $35.68 \%$ \\
\cline { 2 - 6 } & 4 & $13.86 \%$ & $55.06 \%$ & $0.00 \%$ & $31.08 \%$ \\
\hline
\end{tabular}

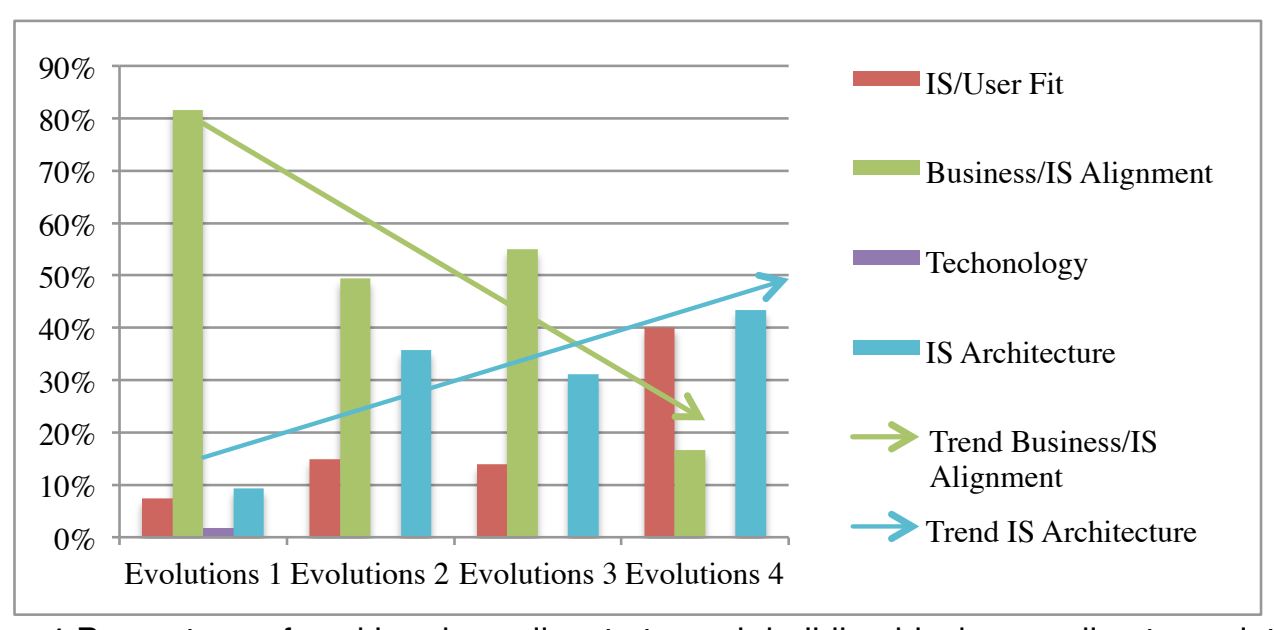

Figure 4.Percentage of working days allocate to each building block according to evolutions 
As shown in Table 3 and graphically in Figure 4 during the first evolutions, the Business/IS Alignment activities are predominant. These evolutions, having a Business/IS Alignment aspect, address only the first level of alignment that is the strategic and operational alignment. This internal level of alignment is an essential and necessary first step to achieve alignment, this can explain the fact that it appears in the first evolutions of the IS.

A second finding is that, over time, a trend to move from activities regarding Business/IS Alignment to activities relating to IS Architecture appears. In other words, after aligning the system, these companies decide to extend the scope of their IS through the integration of new functionalities. However, this trend appears only in company A and company C, consequently further investigations are required to confirm this trend.

So internal alignment seems to be a priority before extending the scope of the IS. Another finding that enforced this trend is the amount of activities related to users. In fact, IS Architecture activities come with user related activities. When a company extends the scope of their IS, in complement, they organize training activities with users.

\section{Discussion}

The results presented here arose from six exploratory case studies in which IS studied did four or less evolutions, so it is clearly a too small number to allow strong generalizations based on them. Indeed, it is not the main purpose of this article, which is to expose the approach to classify evolution and their activities according to the EVOLIS framework.

However, these results points out hypothesis and opportunities for future research. Based on trends highlighted by Figure 4, some questions have a potential research interest:

- Does the proportion of Business/IS Alignment activities decrease over time?

- Does the proportion of activities related to the IS Architecture (namely integration oriented) increase over time?

For the first question, a research opportunity could be to determine why so much time is spent in business/IS alignment in the first evolutions. Is it only related to the recent implementation of the IS, or are there other reasons?

For the second question, as mention previously, the trend to increase activities related to IS architecture appears only in two companies (A and C). Nevertheless, it could be an interesting question to explore.
As you can notice on all tables and figures, we do not compare the global cost of each evolution mainly because the number of IS studied and the number of evolutions is too small to extract valuable results. Nevertheless, the study of the Cost component of the framework could points out interesting results especially the state of the life cycle in which the IS stands.

\section{Conclusion}

This research presents a framework that helps IS managers identify and control the elements triggering an evolution of the system and their consequences. This framework helps managers evaluate and characterize the impact of changes according to 4 blocks of factors influencing IS evolution: IS/Users Fit, Technology, IS Architecture and Business/IS Alignment. The framework encompasses a fifth factor that is the Cost of IS. The strict notion of cost is not addressed by this study but will be subject of future work. Another contribution of this research is that it integrates in the same framework criteria that are usually addressed separately. This enables the design of an evolution scorecard on variables affected by system changes. Moreover, the repeated use of this framework adds a temporal dimension to each criterion and provides a temporal view of system evolution. Furthermore, a temporal view clearly highlights specific evolution patterns of the IS. This view can help managers in designing a strategy of IS evolution by indicating in which direction the system should/could evolve.

The cases analysis produced several results. First it provides a classification method to analyze evolution records in a systematic way in order to provide comparable results for benchmarking IS evolutions in their system life cycle. Second it confirms that grouping variables covering 4 domains (framework 4 factors influencing IS evolution) produces interesting results about the observed systems evolution.

As the activities and evolution classification process remains quite simple, we believe that a third person can reach a similar classification with a good explanation of the framework and the classification process.

Further research could enhance this method to allow systematic extraction of evolution records from very large set of data. This systematic extraction could be assisted by more automatic tools/scripts and so on. The study of large records sets could confirm the typical evolution patterns related to the criteria of our framework. A good knowledge of these patterns is a useful tool for IS managers in charge of designing an evolution strategy for their systems. 
Another opportunity for further research is to determine why so much time is spent in business/IS alignment in the first years of using an IS.

\section{References}

[1]A. Métrailler and T. Estier, "EVOLIS: A Framework for Evaluating Evolution of Information Systems," in Actes $d u$ Colloque AIM 2012, 2012.

[2]M. M. Lehman and J. F. Ramil, "Rules and tools for software evolution planning and management," Proceedings of the 4th international workshop on Principles of software evolution, vol. 11, no. 1, pp. 70-74, 2001.

[3] M. M. Lehman, "Programs, life cycles, and laws of software evolution," Proceedings of the IEEE, vol. 68, no. 9, pp. 1060-1076, 1980.

[4]B. P. Lientz and E. B. Swanson, Software maintenance management: a study of the maintenance of computer application software in 487 data processing organizations, vol. 4, no. 7. Addison-Wesley Reading MA, 1980, p. 294.

[5]N. Chapin, J. E. Hale, K. M. Khan, J. F. Ramil, and W.-G. Tan, "Types of software evolution and software maintenance," Journal of Software Maintenance and Evolution: Research and Practice, vol. 13, no. 1, pp. 3-30, Jan. 2001

[6]J. Buckley, T. Mens, M. Zenger, A. Rashid, and G. Kniesel, "Towards a taxonomy of software change," Journal of Software Maintenance and Evolution: Research and Practice, vol. 17, no. 5, pp. 309-332, Sep. 2005.

[7]K. Lyytinen and M. Newman, "Explaining information systems change: a punctuated socio-technical change model," European Journal of Information Systems, vol. 17, no. 6, pp. 589-613, Dec. 2008.

[8]A. R. Hevner, S. T. March, J. Park, and S. Ram, "Design science in information systems research," Management information systems quarterly, vol. 28, no. 1, pp. 75-106, 2004.

[9]B. Kuechler and V. Vaishnavi, "On theory development in design science research: anatomy of a research project," European Journal of Information Systems, vol. 17, no. 5, pp. 489-504, 2008.

[10] I. Benbasat, D. Goldstein, and M. Mead, "The case research strategy in studies of information systems," MIS quarterly, vol. 11, no. 3, pp. 369-386, 1987.
[11] G. Camponovo and Y. Pigneur, "Information systems alignment in uncertain environments," Proceedings of Decision Support Systems, pp. 134-146, 2004.

[12] Y. E. Chan, S. L. Huff, D. W. Barclay, and D. G. Copeland, "Business Strategic Orientation, Information Systems Strategic Orientation," Information systems research, vol. 8, no. 2, p. 125, 1997.

[13] R. From, I. B. M. Systems, J. C. Henderson, and N. Venkatraman, "Strategic alignment: Leveraging information technology for transforming organizations," IBM Systems Journal, vol. 32, no. 1, pp. 472-484, 1993.

[14] R. Sabherwal and R. Hirschheim, "The dynamics of alignment: Insights from a punctuated equilibrium model," Organization Science, vol. 12, no. 2, pp. 179-197, 2001.

[15] H. Tuan, K. Vo, and H. Elsner, "Management of Portal Evolution - Introducing Evolution Management for the Corporate Financial Portal," in Wirtschaftsinformatik Proceedings, eOrganisation: Service-, Prozess-, MarketEngineering Vol. 2, 2007, pp. 337-352.

[16] B. F. D. Davis, "Information Technology Perceived Usefulness and Perceived Ease of Use," MIS Quarterly, no. September, pp. 319-339, 1989.

[17] A. K. M. N. Islam, M. Koivulahti-Ojala, and T. Käkölä, "A lightweight, industrially-validated instrument to measure user satisfaction and service quality experienced by the users of a UML modeling tool," in AMCIS 2010 Proceedings, 2010 .

[18] A. W. Brown, D. J. Carney, E. J. Morris, D. B. Smith, and P. F. Zarrella, Principles of CASE tool integration. Oxford University Press, USA, 1994.

[19] J. a. Zachman, "A framework for information systems architecture," IBM Systems Journal, vol. 38, no. 2, pp. 454470, 1999.

[20] K. Milis and R. Mercken, "The use of the balanced scorecard for the evaluation of information and communication technology projects," International Journal of Project Management, vol. 22, no. 2, pp. 87-97, 2004.

[21] R. K. Yin, Case Study Research: Design and Methods, vol. 5, no. 5. Sage Publications, 2009, p. 219.

[22] G. Paré and L. Dubé, "Rigor in information systems positivist case research: current practices, trends, and recommendations1," MIS Quarterly, vol. 27, no. 4, pp. 597635, 2003. 